\title{
Influence of scan body design and digital implant analogs on implant replica position in additively manufactured casts
}

\author{
Revilla-León, Marta ; Fogarty, Rachel ; Barrington, Jennifer J ; Zandinejad, Amirali ; Özcan, Mutlu
}

\begin{abstract}
STATEMENT OF PROBLEM Additive manufacturing (AM) technologies can be used to fabricate definitive casts for implant-supported restorations. However, information regarding the accuracy of the implant replica position on the polymeric AM cast generated with different scan bodies and digital implant replica systems is lacking. PURPOSE The purpose of this in vitro study was to compare with a conventional stone cast the linear and angular discrepancies of the implant analog positions in a polymeric AM cast obtained from 3 different scan body and digital implant replica systems. MATERIAL AND METHODS A partially edentulous maxillary typodont with 3 implant replicas (Implant replica RP Branemark system; Nobel Biocare) was prepared. Two duplicating methods were evaluated: conventional (CNV group) and AM (AM group) procedures. For the CNV group, polyvinyl siloxane open-tray implant impressions $(\mathrm{CNV})$ were made at room temperature $\left(23^{\circ} \mathrm{C}\right)$. The AM group was further divided into the subgroups Elos Medtech, Nt-Trading, and Dynamic Abutment. For the Elos Medtech subgroup, the corresponding scan bodies were placed on each implant, and the typodont was digitized by using a laboratory scanner (E3 scanner; 3Shape A/S). The same procedure was repeated with the remaining subgroups. All the AM polymer casts were fabricated at once by using the same 3D printer (Eden $500 \mathrm{~V}$; Stratasys). Ten specimens of each group were obtained $(\mathrm{n}=10)$. A coordinate-measuring machine (CMM) was used to measure the position of each implant replica, and distortion was calculated for each system at the $\mathrm{x}-, \mathrm{y}-$, and $\mathrm{z}$-axes and $3 \mathrm{D}$ distortion measurement $\left(3 \mathrm{D}=\mathrm{x}^{2}+\mathrm{y}^{2}+\mathrm{z}^{2}\right)$. The Shapiro-Wilk test revealed that the data were not normally distributed. The Kruskal-Wallis and pairwise Mann-Whitney U tests $(=.05$ ) were used for the analysis. RESULTS The CNV group presented significantly higher linear discrepancy than the Dynamic Abutment group on the x- and y-axes. On the z-axis, however, the CNV group showed significantly lower linear discrepancy than the Nt-Trading and Dynamic Abutment groups. The 3D linear discrepancy was $12 \pm 12 \mathrm{~m}$ for the CNV group, $4 \pm 100 \mathrm{~m}$ for the Elos Medtech group, $8 \pm 52 \mathrm{~m}$ for the Nt-Trading group, and $5 \pm 19 \mathrm{~m}$ for the Dynamic Abutment. The CNV group demonstrated a significantly higher angle than the Nt-Trading group but a significantly smaller angle than the Elos Medtech and Dynamic Abutment groups. CONCLUSIONS The AM groups had lower 3D discrepancies than the CNV group. The Dynamic Abutment group had significantly better accuracy for the mesiodistal and buccolingual implant replica positions than the CNV group, but the conventional procedures had significantly better results for the apicocoronal implant replica position. Scan body and digital implant replica design systems only influenced the accuracy of the angular implant replica position on the AM casts.
\end{abstract}

DOI: https://doi.org/10.1016/j.prosdent.2019.07.011

Posted at the Zurich Open Repository and Archive, University of Zurich

ZORA URL: https://doi.org/10.5167/uzh-198024

Journal Article

Accepted Version 
Originally published at:

Revilla-León, Marta; Fogarty, Rachel; Barrington, Jennifer J; Zandinejad, Amirali; Özcan, Mutlu (2020). Influence of scan body design and digital implant analogs on implant replica position in additively manufactured casts. Journal of Prosthetic Dentistry, 124(2):202-210.

DOI: https://doi.org/10.1016/j.prosdent.2019.07.011 
Influence of scan body design and digital implant analogs on implant replica position in additively manufactured casts

Marta Revilla-León DDS, MSD, ${ }^{a}$ Rachel Fogarty DDS, ${ }^{\mathrm{b}}$ Jennifer J. Barrington DDS, ${ }^{\mathrm{c}}$ Amirali Zandinejad DDS, MS, ${ }^{\mathrm{d}}$ AND Mutlu Özcan DDS, DMD, PhD

${ }^{a}$ Assistant Faculty and Assistant Program Director AEGD, College of Dentistry, Texas A\&M University, Dallas, Texas; Affiliate Faculty Graduate Prosthodontics University of Washington, Seattle, Wash; Researcher at Revilla Research Center, Madrid, Spain.

${ }^{\mathrm{b}}$ Resident AEGD Program, College of Dentistry, Texas A\&M University, Dallas, Texas.

${ }^{\mathrm{c} C l i n i c a l}$ Associate Professor, Department of General Dentistry, College of Dentistry, Texas A\&M University, Dallas, Texas.

${ }^{\mathrm{d} A s s o c i a t e}$ Faculty and Program Director AEGD, College of Dentistry, Texas A\&M University, Dallas, Texas.

'Professor and Head, Dental Materials Unit, Center for Dental and Oral Medicine, University of Zürich, Switzerland.

Corresponding author:

Dr Marta Revilla-León

3302 Gaston Avenue

Room 713

Dallas, Texas, USA 75246

Email: revillaleon@tamhsc.edu 


\begin{abstract}
Statement of problem. Additive manufacturing (AM) technologies can be used to fabricate definitive casts for implant-supported restorations. However, information regarding the accuracy of the implant replica position on the polymeric AM cast generated with different scan bodies and digital implant replica systems is lacking.
\end{abstract}

Purpose. The purpose of this in vitro study was to compare with a conventional stone cast the linear and angular discrepancies of the implant analog positions in a polymeric AM cast obtained from 3 different scan body and digital implant replica systems.

Material and methods. A partially edentulous maxillary typodont with 3 implant replicas (Implant replica RP Branemark system; Nobel Biocare) was prepared. Two duplicating methods were evaluated: conventional (CNV group) and AM (AM group) procedures. For the CNV group, polyvinyl siloxane open-tray implant impressions (CNV) were made at room temperature $\left(23^{\circ} \mathrm{C}\right)$. The AM group was further divided into the subgroups Elos Medtech, Nt Trading, and Dynamic abutment. For the Elos Medtech subgroup, the corresponding scan bodies were placed on each implant, and the typodont was digitized using a laboratory scanner (E3 scanner; 3Shape). The same procedure was repeated with the remaining subgroups. All the AM polymer casts were fabricated at once using the same 3D printer (Eden 500V; Stratasys). Ten specimens of each group were obtained $(n=10)$. A coordinate measuring machine $(C M M)$ was used to measure the position of each implant replica, and distortion was calculated for each system at the x-, y-, and z-axes and $3 \mathrm{D}$ distortion measurement $\left(3 D=\sqrt{x^{2}+y^{2}+z^{2}}\right)$. The Shapiro-Wilk test revealed that the data were not normally distributed. The Kruskal-Wallis and pairwise Mann-Whitney U tests $(\alpha=.05)$ were used for the analysis. 
Results. The CNV group presented significantly higher linear discrepancy than the Dynamic abutment group on the $\mathrm{x}$ - and $\mathrm{y}$ - axes. On the z-axis, however, the CNV group showed significantly lower linear discrepancy than the Nt-Trading and Dynamic abutment groups. The 3D linear discrepancy was $12 \pm 12 \mu \mathrm{m}$ for the CNV group, $4 \pm 100 \mu \mathrm{m}$ for the Elos Medtech group, $8 \pm 52 \mu \mathrm{m}$ for the Nt-Trading group, and $5 \pm 19 \mu \mathrm{m}$ for the Dynamic abutment. The CNV group demonstrated a significantly higher angle than the Nt-Trading group, but a significantly smaller angle than the Elos Medtech and Dynamic abutment groups.

Conclusions. The AM groups had lower 3D discrepancies than the CNV group. The Dynamic abutment group had significantly better accuracy for the mesiodistal and buccolingual implant replica position compared with that of the CNV group, but the conventional procedures had significantly better results for the apicocoronal implant replica position. Scan body and digital implant replica design systems only influenced the accuracy of the angular implant replica position on the AM casts.

\section{CLINICAL IMPLICATIONS}

The polyjet additive manufacturing technology tested is a reliable method of duplicating implant definitive casts. The 3-scan body and digital implant replica systems obtained better accuracy with the $3 \mathrm{D}$ implant position transference than with the conventional procedures.

\section{INTRODUCTION}

A definitive implant cast should accurately represent the 3D implant position of the patient's mouth in relation to the surrounding intraoral tissues. ${ }^{1-3}$ The accuracy of the conventional implant definitive cast can be affected by factors including the polymerization shrinkage of the 
impression material, ${ }^{4-6}$ the setting expansion of the dental stone, ${ }^{4-6}$ the number of implants present,${ }^{3}$ the implant angulation, ${ }^{3,7}$ whether a splinted or nonsplinted implant impression technique was used, ${ }^{3,8-19}$ and the design and rigidity of the impression tray. ${ }^{4-6}$

The incorporation of intraoral scanners (IOSs) has provided a technique for digitizing the clinical situation, including the 3D position of an implant. ${ }^{20-22}$ As with conventional impressions, a scan body must be positioned on each implant to transport the 3D position of each implant into the computer-aided design (CAD) software. The outcome of such a digital scan is a standard tessellation language (STL) file that represents the virtual implant definitive cast with the implant position coordinates on the $\mathrm{x}-, \mathrm{y}-$, and $\mathrm{z}$-axes. $^{23}$

Previous studies have evaluated the accuracy of the IOSs when scanning implants. ${ }^{13-20,24-}$ ${ }^{30}$ Factors that may influence the accuracy of the digital scan include implant angulation, ${ }^{25}$ distance between the implants, ${ }^{27}$ scan body design, ${ }^{31,32}$ scanning protocol, $, 3,34$ calibration of the intraoral digitizer device, ${ }^{35}$ handling and learning, ${ }^{36,37}$ and ambient scanning light conditions. ${ }^{38-40}$ Furthermore, scanning accuracy differences based on the different scanning technologies should be expected..$^{41-50}$

Additive manufacturing (AM) technologies have allowed the integration of digitizing procedures using an IOS into the fabrication of definitive casts. A definitive cast can be obtained from an STL file with AM technology. The accuracy of AM technologies for duplicating conventional implant definitive casts has been evaluated by using a coordinate measuring machine $(\mathrm{CMM}){ }^{51}$ The authors reported higher accuracy of the implant replica positions on the AM duplicated casts compared with duplicated casts using conventional procedures. ${ }^{51}$ However, the influence of the scan body and digital implant replica designs on the accuracy of the digital implant replica positions on the AM definitive cast remains unclear. 
The purpose of the present in vitro study was to compare the linear and angular discrepancies of the implant replica positions on the polymeric AM cast obtained from 3 different scan body and digital implant replica systems with a conventional stone cast. The null hypotheses were that no significant difference in the linear and angular discrepancies on the implant replica positions would be found between the conventional and AM duplicated implant definitive casts and that no significant difference in the linear and angular discrepancies on the implant replica positions would be found between the different AM duplicated implant definitive casts.

\section{MATERIAL AND METHODS}

A maxillary partially dentate typodont (Hard gingiva jaw model MIS2009-U-HD-M-32; Nissin) was used. The right first molar, right first and second premolar, and left second premolar denture teeth were present (Fig. 1). Three implant replicas (3 Implant replica RP Brånemark system; Nobel Biocare) were placed in the edentulous areas at the right and left canines and second left premolar positions and secured with acrylic resin (Pattern resin; GC America). The 3-mm most coronal part of the implant replicas was covered with tissue moulage (Soft tissue moulage; Kerr Corp) to simulate clinical conditions and facilitate the posterior measurements (Fig. 1). The maxillary typodont was then duplicated using 2 methodologies: conventional procedures (CNV group) and a polyjet AM technology (AM group).

For the fabrication of the CNV specimen, an implant impression abutment (Impression coping open tray Brånemark system RP; Nobel Biocare) was positioned and tightened to $15 \mathrm{Ncm}$ using a torque wrench (Manual torque wrench prosthetic; Nobel Biocare) on each implant replica of the maxillary typodont. An impression with a custom tray and addition polyvinyl siloxane 
impression material (Virtual heavy and light viscosity regular set; Ivoclar Vivadent AG) was made at room temperature $\left(23^{\circ} \mathrm{C}\right)$. After the complete polymerization of the impression material, the impression was recovered. An implant replica (Implant replica Brånemark system RP; Nobel Biocare) was positioned on each implant impression abutment and tightened to $15 \mathrm{Ncm}$ using the same torque wrench. The 3-mm most coronal part of the implant replicas was covered with tissue moulage (Soft tissue moulage; Kerr Corp). The impression was poured with Type IV dental stone (GC Fujirock EP; GC America) after mixing $22 \mathrm{~mL}$ water with $110 \mathrm{~g}$ dental stone under vacuum for 30 seconds. The cast was recovered after the dental stone had completely set (Fig. 2). The procedure was repeated until 10 specimens were obtained.

Three AM subgroups were created based on the intraoral scan body and digital implant replica systems evaluated (Table 1): AM-1 (ELOS Medtech), AM-2 group (Nt-trading), and AM-3 (Dynamic Abutment). For the AM-1 specimen fabrication, an intraoral scan body (Elos accurate IO scan body Brånemark system RP; ELOS Medtech) (Fig. 3A) was hand-tightened on each implant replica on the maxillary typodont. Then, the typodont was digitized using a laboratory scanner (E3 scanner; 3Shape) at a constant room temperature of $23{ }^{\circ} \mathrm{C}$. The scanner was calibrated before the procedure by following the manufacturer's recommendations. Dental CAD software (Model Builder, Dental System; 3Shape) was used to create the implant definitive cast with the correspondent implant replicas (Elos accurate model analog Brånemark system RP; ELOS Medtech) library of the software. The STLAM1 was exported, and the same procedure was repeated for each AM-2 (Fig. 3B) and AM-3 (Fig. 3C) with the specific scan bodies (Table 1). All the scan bodies were new.

The STL $\mathrm{AM}_{\mathrm{A} 1}$, STL $\mathrm{AM} 2$, and STL $\mathrm{SM}_{\mathrm{A} 3}$ were used to fabricate the polymeric AM casts with a polyjet AM technology and a 3D printer (Eden 500V; Stratasys). In order to calibrate the 
procedure, all the polymeric (VeroDent MED670; Stratasys) AM specimens (AM-1, AM-2, and AM-3) were fabricated at the same time in the selected 3D printer at a constant room temperature of $23{ }^{\circ} \mathrm{C}$ (Figs. 4-6). A new digital implant replica was positioned on each corresponding housing of each implant definitive cast after manufacturing the casts of the AM groups (Fig. 4).

A CMM was used to evaluate the position of the implant replicas on the $\mathrm{x}_{-}, \mathrm{y}-$, and $\mathrm{z}-$ axes. The position of the center point of all the implant replicas was measured with the CMM (CMM Contura G2 10/16/06 RDS; Carl Zeiss Industrielle Messtechnik GmbH) in an independent laboratory (LID S.L.). The nominal linear accuracy of the machine was described by the manufacturer to be within $1 \mu \mathrm{m}$ in all axes. The implant replica positions of the maxillary typodont were measured and used as a reference (control) to compare the 40 different casts. The position of both the maxillary right and left canines implant replicas had 8.9 degrees of divergence.

A 0.5-mm stylus (SensorVast XXT $0.5 \mathrm{~mm}$; Carl Zeiss Industrielle Messtechnik GmbH) scanning head under a light force of $0.1 \mathrm{~N}$ was used to measure the position center point of each implant replica in 3 linear and angular dimensions by using the $\mathrm{x}-$, $\mathrm{y}-$, and $\mathrm{z}$-axes. After the measurements of the implant replica positions of the maxillary typodont and all the specimens, the data of the center points of the implant replicas were analyzed for the 3 dimensional linear and angular discrepancies between each implant replica and the corresponding implant replica of the typodont using the best-fit technique in a CAD software (Geomagic; 3D Systems). The 3D linear distortion was calculated using the formula $3 D=\sqrt{x^{2}+y^{2}+z^{2}}$. For the angular discrepancies' calculations, the axis of each implant replica of the typodont was calculated. Each axis has 2 projections, one on the $\mathrm{X}$-axis ( $\mathrm{XZ}$ angle) and the other on the $\mathrm{y}$-axis (YZ angle). The distortion between the axis of each implant replica of the typodont and the axis of each implant 
replica of each specimen was calculated using the best fit technique in the CAD software (Geomagic; 3D Systems).

The Shapiro-Wilk test revealed that the data were not normally distributed. Therefore, the linear $(\mu \mathrm{m})$ and angular discrepancies (degrees) were analyzed using the Kruskal-Wallis test, followed by pairwise Mann-Whitney $U$ tests between the CNV and AM groups $(\alpha=.05)$.

Statistical analysis was performed with statistical software (IBM SPSS Statistics, v20; IBM Corp).

\section{RESULTS}

The Kruskal-Wallis test revealed a significant difference in linear and angular discrepancies for specimens duplicated using conventional and AM approaches $(P<.05)$. To determine the significance of pairwise differences between the CNV and AM groups, the data were analyzed using the Mann-Whitney U tests subjected to Bonferroni corrections (.05/6) for pairwise comparisons (adjusted $\alpha=.008$ ) (Table 2).

The CNV group presented significantly higher linear discrepancy than the AM-3 group on the $\mathrm{x}$ - and $\mathrm{y}$-axes. On the z-axis, however, the CNV group showed significantly lower linear discrepancy than the AM-2 and AM-3 groups (Figs. 7 and 8). Furthermore, no significant differences were found in the linear discrepancies on the x-, y-, and z-axes between the different AM groups. The 3D linear discrepancy was $12 \pm 12 \mu \mathrm{m}$ for the CNV group, $4 \pm 100 \mu \mathrm{m}$ for the AM-1 group, $8 \pm 52 \mu \mathrm{m}$ for the AM-2 group, and $5 \pm 19 \mu \mathrm{m}$ for the AM-3 group.

The pattern of variability for angle XZ was inconsistent, with the CNV group demonstrating a significantly higher angle than that of the AM-2 group, but a significantly smaller angle than for the AM-1 and AM-3 groups. Furthermore, XZ angular discrepancy for the 
AM-2 group was significantly lower than that of the AM-1 and AM-3 groups. Angle YZ for AM3 was significantly higher than for the control and AM2 groups.

\section{DISCUSSION}

Significant linear and angular discrepancies were encountered among the CNV and AM groups, and significant angular discrepancies were found among the different AM groups. Therefore, both null hypotheses were rejected.

An open-tray implant impression technique was used to fabricate the specimens for the CNV group. The accuracy of splinting and nonsplinting implant impression techniques has been assessed when 2 implants with different degrees of divergence were present in a partially edentulous patient, with results supporting the technique of splinting the implant impression. ${ }^{3,11}$ However, when the implants were parallel or had less than 10 degrees of divergence, the results were not consistent. Authors reported higher, ${ }^{11,14}$ no difference, ${ }^{10,12,13}$ or decreased ${ }^{16-18}$ impression accuracy with the nonsplinting technique. Because of the implant distribution between the denture teeth of the maxillary typodont used, only 2 implants with less than 10 degrees of divergence could have been splinted with the implant impression procedure.

The thermal contraction of the impression material, because of the different temperature between the impression procedure in the patient's mouth $\left(37^{\circ} \mathrm{C}\right)$ and the impression pouring at room temperature $\left(23^{\circ} \mathrm{C}\right)$, has been reported, ${ }^{52}$ which could lead to a dimensional change between 40 to $52 \mu \mathrm{m}$ in the anterior region and between 68 to $88 \mu \mathrm{m}$ in the posterior region. ${ }^{52} \mathrm{In}$ the present study, all the conventional procedures were performed at room temperature, which did not model the volumetric shrinkage of the impression material encountered clinically, ${ }^{52}$ producing therefore a more accurate impression technique. 
A laboratory dental scanner was used to digitize the typodont for fabrication of the AM specimens as higher scanning accuracy compared with that of IOSs has been reported, ${ }^{53-55}$ minimizing errors including the scanning protocol, ${ }^{33,34}$ ambient light scanning conditions, ${ }^{38-40}$ and handling and learning. ${ }^{36,37}$ The goal of the present study was to evaluate the impact of the scan body and implant replica designs on the digital implant replica position accuracy on the AM casts. Extrapolation of the results should be made carefully if an IOS is used as a digitizing device.

Based on the results of the present study, the polyjet AM technology tested was a reliable duplicating method for implant definitive casts as AM groups presented lower 3D discrepancies compared with those of the CNV group. Furthermore, the CNV group presented significantly higher linear discrepancy than the AM-3 group in the $\mathrm{x}$ - and $\mathrm{y}$-axes. In the $\mathrm{z}$-axis, however, the CNV group showed significantly lower linear discrepancy than the AM-2 and AM-3 groups. The boxplot (Fig. 7) representing the positive and negative discrepancies values on the $\mathrm{x}-, \mathrm{y}-$, and $\mathrm{z}-$ axis indicated a more mesiodistal, buccolingual, or apicocoronal position of an implant replica compared with the implant replica of the typodont. In all groups, the implant replicas showed higher accuracy in the apicocoronal position compared with the mesiodistal and buccolingual positions The broad ranges from a positive to a negative value provide a high absolute mean and standard deviation values.

The differences in the results of the present in vitro study among the AM groups could not differentiate among the stages of digital workflow, digitizing, or manufacturing phases as to higher distortion. The goal of the present study was to analyze the capability to transfer the 3D implant position from the digitizing procedures of the typodont using 3 scan body and implant replica systems and a laboratory scanner to the polyjet AM implant definitive cast. Because the 
same data capturing procedure and polyjet 3D printer were used to manufacture all the $\mathrm{AM}$ specimens, the design of the scan body and digital implant replica systems influenced only the angular implant replica position accuracy on the AM casts.

When designing the printing strategy of an AM cast, different build orientation can be selected. The characteristically superficial texture consequence of the layer-by-layer building procedure and its relationship with the implant replica angulation may also influence the digital implant replica insertion and position on the AM cast (Fig. 9). In the present study, all the AM implant definitive casts were fabricated at once in the 3D printer with the same printing strategy and perpendicular to the building platform. All the implant replicas were new, positioned on the AM specimen only once, and consecutively measured.

The resiliency or deformation of the polymer when the implant replica was placed on the AM specimen may have influenced the position of the implant replicas in the AM implant definitive cast. The implant replicas had 2 different positioning systems: pressure and screwretained. For the AM-1 group, the digital implant replica was placed with hand-pressure until its apicocoronal stop design touched the apicocoronal stop of the housing on the AM cast (Fig. 9). However, the AM-2 and AM-3 specimens consisted of 2-piece screw-retained implant replicas, where once the coronal part of the implant replica was positioned into the AM cast, the apical part of the implant replica was screw-retained into the coronal one. Based on the results of the present study, discrepancies were greater with the 2-piece screw-retained implant replicas than the pressure positioned digital implant replica. Furthermore, the AM-1 group had similar accuracy for the apicocoronal position of an implant compared with conventional procedures.

The accuracy of the AM technologies as a duplicating method of the conventional implant definitive casts has been measured using a CMM. ${ }^{40} \mathrm{~A}$ completely edentulous 
conventional stone cast with 7 implant replicas (Tissue Level RN Straumann Implant Replica; Straumann) was duplicated using conventional and AM procedures. The authors reported even higher accuracy in the implant replica positions on the AM duplicated casts compared with duplicated casts using conventional procedures. However, comparisons with the present study are difficult because of the different AM technologies used and the use of only 1 design for the scan bodies and digital implant replica.

Limitations of the present study include possible variation of implant angulation and depth, splinting and nonsplinting implant impression techniques, different scan body and implant replica designs, and the use of a laboratory scanner as the digitizer system. Furthermore, in vitro and clinical studies are recommended to assess the impact of different angulations, depths, implant connections, different IOSs and digitizing variables, 3D printing build orientation, postprocessing procedures, variability of polymers available for manufacture with AM technologies, and AM technologies available for processing polymer dental casts.

\section{CONCLUSIONS}

Based on the findings of this in vitro study, the following conclusions were drawn:

1. The polyjet additive manufacturing technology evaluated is a reliable duplicating method for implant definitive casts. The lower overall 3D discrepancy was obtained in the Elos Medtech group, followed by the Dynamic abutment, by the Nt-Trading group, and by the CNV group.

2. The AM-3 group had significantly better accuracy on the mesiodistal and buccolingual implant replica position compared with the conventional group, but conventional procedures had significantly better results for the apicocoronal implant replica position. 
3. No significant differences were measured on the linear discrepancies among the different AM groups. The pattern of variability for angle XZ was inconsistent, and the CNV group obtained a significantly higher angle than Nt-trading, but a significantly smaller angle than the Elos Medtech and Dynamic abutment groups. Furthermore, XZ angular discrepancy for Nt-trading was significantly lower than for other AM groups. Angle YZ for the Dynamic abutment was significantly higher for the control and Nt-Trading groups.

4. The 3-scan body and digital implant replica systems evaluated obtained better accuracy of the 3D implant position transference compared with conventional procedures. 


\section{REFERENCES}

1. Rosenstiel SF, Land MF, Fujimoto J. Contemporary fixed prosthodontics. 5th ed. St. Louis: Mosby/Elsevier; 2016. p. 369-77.

2. Ortop A, Jemt T, Bläck T, Jälevik T. Comparisons of precision of fit between cast and CNCmilled titanium implant frameworks for the edentulous mandible. Int J Prosthodont 2003;16:194200.

3. Lee H, So JS, Hochstedler JL, Ercoli C. The accuracy of implant impressions: A systematic review. J Prosthet Dent 2008;100:285-91.

4. Reisbick MH, Matyas J. The accuracy of highly filled elastomeric impression materials. J Prosthet Dent 1975;33:67-72.

5. McCabe JF, Storer R. Elastomeric impression materials. The measurement of some properties relevant to clinical practice. Br Dent J 1980;73:73-9.

6. Ciesco JN, Malone WFP, Sandrik JL, Mazur B. Comparison of elastomeric impression materials used in fixed prosthodontics. J Prosthet Dent 1981;45:89-94.

7. Jaffin R, Berman C. The excessive loss of Brånemark implants in type IV bone: a 5-year analysis. J Periodontol 1991;62:2-4.

8. Mojon P, Oberholzer JP, Meyer JM, Belser UC. Polymerization shrinkage of index and pattern acrylic resins. J Prosthet Dent 1990;64:684-8.

9. Spector MR, Donovan TE, Nicholls JI. An evaluation of impression techniques for osseointegrated implants. J Prosthet Dent 1990;63:444-7.

10. Carr AB. Comparison of impression techniques for a two-implant 15-degree divergent model. Int J Oral Maxillofac Implants 1992;7:468-75. 
11. Assunção WG, Filho HG, Zaniquelli O. Evaluation of transfer impressions for osseointegrated implants at various angulations. Implant Dent 2004;13:358-66.

12. Choi JH, Lim YJ, Yim SH, Kim CW. Evaluation of the accuracy of implant-level impression techniques for internal-connection implant prostheses in parallel and divergent models. Int J Oral Maxillofac Implants 2007;22:761-8.

13. Lee YJ, Heo SJ, Koak Jy, Kim SK. Accuracy of different impression techniques for internalconnection implants. Int J Oral Maxillofac Implants 2009;24:823-30.

14. Sorrentino R, Gherlone EF, Calesini G, Zarone F. Effect of implant angulation, connection length, and impression material on the dimensional accuracy of implant impressions: An in vitro compara- tive study. Clin Implant Dent Relat Res 2010;12:e63-76.

15. Papaspyridakos P, Benic GI, Hogsett VL, White GS, Lal K, Gallucci GO. Accuracy of implant casts generated with splinted and nonsplinted impression techniques for edentulous patients: an optical scanning study. Clin Oral Impl Res 2012;23:676-81.

16. Rutkunas V, Sveikata K, Savickas R. Effects of implant angulation, material selection, and impression technique on impression accuracy: A preliminary laboratory study. Int J Prosthodont $2012: 25: 512-5$

17. Tarib NA, Seong TW, Chuen KM, Kun MS, Ahmad M, Kamarudin KH. Evaluation of splinting implant impression techniques: Two dimensional analyses. Eur J Prosthodont Restor Dent 2012;20:35-9.

18. Al-Abdullah K, Zandparsa R, Finkelman M, Hirayama H. An in vitro comparison of the accuracy of implant impressions with coded healing abutments and different implant angulations. J Prosthet Dent 2013;110:90-100. 
19. Papaspyridakos P, Chen CJ, Gallucci GO, Doukoudakis A, Weber HP, Chronopoulos V. Accuracy of implant impressions for partially and completely edentulous patients: a systematic review. Int J Oral Maxillofac Implants 2014;29:836-45.

20. Al-Jubuori O, Azari A. An introduction to dental digitizers in dentistry. A systematic review. J Chem Pharm Res 2015;7:10-20.

21. Moreira AH, Rodrigues NF, Pinho ACM, Fonseca JC, Vilaça JL. Accuracy comparison of implant impression techniques: a systematic review. Clin Imp Dent and Relat Res 2015;17:e75164.

22. Rutkūnas V, Gečiauskaitė A, Jegelevičius D, Vaitiekūnas M. Accuracy of digital implant impressions with intraoral scanners. A systematic review. Eur J Oral Implantol 2017;0:101-20. 23. Hull CW. Apparatus for production of three-dimensional objects by stereolithography, US Patent 4575330, 1986.

24. Chew AA, Esguerra RJ, Teoh KH, Wong KM, Ng SD, Tan KB. Three-dimensional accuracy of digital implant impressions: Effect of different scanners and implant level. Int J Oral Maxillofac Implants 2016;32:70-80.

25. Gimenez-Gonzalez B, Hassan B, Özcan M, Pradíes G. An in vitro study of factors influencing the performance of digital intraoral impressions operating on active wavefront sampling technology with multiple implants in the edentulous maxilla. J Prosthodont 2017;26:650-5.

26. Amin S, Weber HP, Finkelman M, El Rafie K, Kudara Y, Papaspyridakos P. Digital vs. conventional full-arch implant impressions: a comparative study. Clin Oral Implants Res 2017;28:1360-7. 
27. Tan MY, Yee SHX, Wong KM, Tan YH, Tan KBC. Comparison of three-dimensional accuracy of digital and conventional implant impressions: Effect of interimplant distance in an edentulous arch. Int J Oral Maxillofac Implants 2019;34:366-80.

28. Flügge T, Van der Meer W, Gonzalez BG, Vach K, Wismeijer D, Wang P. The accuracy of different dental impression techniques for implant-supported dental prostheses: A systematic review and meta-analysis. Clin Oral Implants Res 2018;29:374-92.

29. Alikhasi M, Siadat H, Nasirpour A, Hasanzade M. Three-dimensional accuracy of digital impression versus conventional method: Effect of implant angulation and connection type. Int $\mathbf{J}$ Dent 2018;2018:3761750.

30. Pesce P, Pera F, Setti P, Menini M. Precision and accuracy of a digital impression scanner in full-arch implant rehabilitation. Int J Prosthodont 2018;31:171-5.

31. Stimmelmayr M, Güth JF, Erdelt K, Edelhoff D, Beuer F. Digital evaluation of the reproducibility of implant scanbody fit-an in vitro study. Clin Oral Investig 2012;16:851-6.

32. Mizumoto RM, Yilmaz B. Intraoral scan bodies in implant dentistry: A systematic review. J Prosthet Dent 2018;120:343-52.

33. Müller P, Ender A, Joda T, Katsoulis J. Impact of digital intraoral scan strategies on the impression accuracy using the TRIOS pod scanner. Quintessence Int 2016;47:343-9.

34. Anh JW, Park JM, Chun YS, Kim M, Kim M. A comparison of the precision of threedimensional images acquired by two intraoral scanners: effects on tooth irregularities and scanning direction. Korean J Orthod 2016;46:3-12.

35. Richert R, Goujat A, Venet L, Viguie G, Viennot S, Robinson P, et al. Intraoral scanners technologies: A review to make a successful impression. J Healthc Eng 2017;1-9. 
36. Kim J, Park JM, Kim M, Heo SJ, Shin IH, Kim M. Comparison of experience curves between two 3-dimensional intraoral scanners. J Prosthet Dent 2016;116:221-30.

37. Lim JH, Park JM, Kim M, Heo SJ, Myung JY. Comparison of digital intraoral scanner reproducibility and image trueness considering repetitive experience. J Prosthet Dent 2018;119:225-32.

38. Arakida T, Kanazawa M, Iwaki M, Suzuki T, Minakuchi S. Evaluating the influence of ambient light on scanning trueness, precision, and time of intra oral scanner. J Prosthodont Res 2018;62:324-9.

39. Revilla-León M, Liang P, Sadeghpour M, Piedra-Cascón W, Zandinejad A, Özcan M, et al. Intraoral digital impressions. Part-1: Influence of ambient scanning light conditions on the accuracy (trueness and precision) of different intraoral scanners. J Prosthet Dent 2019 [Epub ahead of print].

40. Revilla-León M, Liang P, Sadeghpour M, Piedra-Cascón W, Zandinejad A, Özcan M, et al. Intraoral digital impressions. Part-2: Influence of ambient scanning light conditions on the mesh quality of different intraoral scanners. J Prosthet Dent 2019 [Epub ahead of print].

41. Patzelt SB, Vonau S, Stampf S, Att W. Assessing the feasibility and accuracy of digitizing edentulous jaws. J Am Dent Assoc 2013;144:914-20.

42. Patzelt SB, Emmanouilidi A, Stampf S, Strub JR, Att W. Accuracy of full-arch scans using intraoral scanners. Clin Oral Investig 2014;18:1687-94.

43. Joda Joda T, Zarone F, Ferrari M. The complete digital workflow in fixed prosthodontics: a systematic review. BMC Oral Health 2017;17:124-31. 
44. Renne W, Ludlow M, Fryml J, Schurch Z, Mennito A, Kessler R, et al. Evaluation of the accuracy of 7 intraoral scanners: An in vitro analysis based on 3-dimensional comparison. J Prosthet Dent 2017;118:36-42.

45. Khraishi H, Duane B. Evidence for use of intraoral scanners under clinical conditions for obtaining full-arch digital impressions is insufficient. Evid Based Dent 2017;18:24-5.

46. Ahlholm P, Sipilä K, Vallittu P, Jakonen M, Kotiranta U. Digital versus conventional impressions in fixed prosthodontics: A review. J Prosthodont 2018;27:35-41.

47. Medina-Sotomayor P, Pascual-Moscardó A, Camps I. Relationship between resolution and accuracy of four intraoral scanners in complete-arch impressions. J Clin Exp Dent 2018;10:e3616.

48. Abduo J, Elseyoufi M. Accuracy of intraoral scanners: A systematic review of influencing factors. Eur J Prosthodont Restor Dent 2018;26:101-21.

49. Takeuchi Y, Koizumi H, Furuchi M, Sato Y, Ohkubo C, Matsumura H. Use of digital impression systems with intraoral scanners for fabricating restorations and fixed dental prostheses. J Oral Sci 2018;60:1-7.

50. Mennito AS, Evans ZP, Lauer AW, Patel RB, Ludlow ME, Renne WG. Evaluation of the effect scan pattern has on the trueness and precision of six intraoral digital impression systems. $\mathrm{J}$ Esthet Restor Dent 2018;30:113-8.

51. Revilla-León M, Gonzalez-Martín Ó, Pérez López J, Sánchez-Rubio JL, Özcan M. Position accuracy of implant analogs on 3D printed polymer versus conventional dental stone casts measured using a coordinate measuring machine. J Prosthodont 2018;27:560-7.

52. Kim KM, Lee JS, Kim KN, Shin SW. Dimensional changes of dental impression materials by thermal changes. J Biomed Mater Res 2001;58:217-20. 
53. Persson M, Andersson M, Bergman B. The accuracy of a high- precision digitizer for CAD/CAM of crowns. J Prosthet Dent 1995;74:223-9.

54. DeLong R, Heinzen M, Hodges JS, Ko CC, Douglas WH. Accuracy of a system for creating 3D computer models of dental arches. J Dent Res 2003;82:438-42.

55. Del Corso M, Aba G, Vazquez L, Dargaud J, Dohan Ehrenfest DM. Optical threedimensional scanning acquisition of the position of osseointegrated implants: an in vitro study to determine method accuracy and operational feasibility. Clin Implant Dent Relat Res 2009;11:214-21. 


\section{TABLES}

Table 1. Characteristics of implant impression abutment, scan body, and implant replica systems evaluated

\begin{tabular}{|c|c|c|c|c|}
\hline GROUP & $\begin{array}{l}\text { Abutment for implant } \\
\text { position transfer }\end{array}$ & Impression & Implant replica & Cast \\
\hline $\begin{array}{l}\text { CNV } \\
\text { Conventional }\end{array}$ & $\begin{array}{l}\text { Implant abutment } \\
\text { impression } \\
\text { Brånemark system RP; } \\
\text { Nobel Biocare }\end{array}$ & $\begin{array}{l}\text { Elastomeric } \\
\text { impression } \\
\text { Open custom tray }\end{array}$ & $\begin{array}{l}\text { Implant replica RP } \\
\text { Brånemark system; } \\
\text { Nobel Biocare }\end{array}$ & $\begin{array}{l}\text { Type IV dental } \\
\text { stone }\end{array}$ \\
\hline $\begin{array}{l}\text { AM-1 } \\
\text { Elos Medtech }\end{array}$ & $\begin{array}{l}\text { Elos Accurate Intraoral } \\
\text { Scan body } \\
\text { Titanium base, PEEK }\end{array}$ & E3 laboratory & $\begin{array}{l}\text { Elos Accurate Model } \\
\text { Analog }\end{array}$ & $\begin{array}{l} \\
\text { Polymer } \\
\text { (VeroDent } \\
\text { MED670; }\end{array}$ \\
\hline $\begin{array}{l}\text { AM-2 } \\
\text { Nt Trading }\end{array}$ & $\begin{array}{l}\text { 3D Guide Intraoral Scan } \\
\text { body } \\
\text { Titanium base, PEEK }\end{array}$ & $\begin{array}{l}\text { Model Builder, } \\
\text { Dental system } \\
\text { (3Shape) }\end{array}$ & $\begin{array}{l}\text { Nt-Trading digital } \\
\text { implant model (DIM) } \\
\text { analog. } \\
\text { Two-pieces } \\
\text { repositionable } \\
\text { implant replica } \\
\text { (screwed fastening). }\end{array}$ & $\begin{array}{l}\text { Stratasys) } \\
\text { Polyjet AM } \\
\text { (Eden 500V; } \\
\text { Stratasys) }\end{array}$ \\
\hline AM-3 & Intraoral Scan body & & Digital analog. & \\
\hline
\end{tabular}




\begin{tabular}{|l|l|l|l|l|}
\hline Dynamic & system with intraoral & & Two-pieces & \\
Abutment & adaptor. & & repositionable \\
(Talladium) & Connecting element & & (screwed fastening). & \\
& between scan body and & & & \\
implant. & & & & \\
& PEEK scan body. & & & \\
\hline
\end{tabular}

PEEK, polyetheretherketone. 
Table 2. Data analyzed using Mann-Whitney U tests with Bonferroni correction $(\alpha=.008)$

\begin{tabular}{|l|l|l|l|l|l|}
\hline Group & $\mathbf{x}$-axis & $\mathbf{y}$-axis & z-axis & Angle XZ & Angle YZ \\
\hline CNV versus & .308 & .201 & .320 & $<.001^{*}$ & .704 \\
\hline CNV versus & .033 & .233 & $.003^{*}$ & $<.001^{*}$ & .326 \\
\hline AM-2 & $<.001^{*}$ & $<.001^{*}$ & $.001^{*}$ & $<.001^{*}$ & $<.001^{*}$ \\
\hline ANV versus & .616 & .470 & .093 & $<.001^{*}$ & .818 \\
\hline AM-1 versus & AM-2 & .009 & .061 & .021 & .315 \\
\hline AM-1 versus & .483 & .009 & .663 & $<.001^{*}$ & $.004^{*}$ \\
\hline AM-3 & .922 & & & & \\
\hline AM-2 versus & & & & & \\
\hline
\end{tabular}

*Indicates significant difference at $P<.008$. 


\section{FIGURES}

Figure 1. Maxillary partially dentate typodont with 3 implant replicas (Implant replica RP

Brånemark system; Nobel Biocare) positioned on right and left canines and second left premolar.

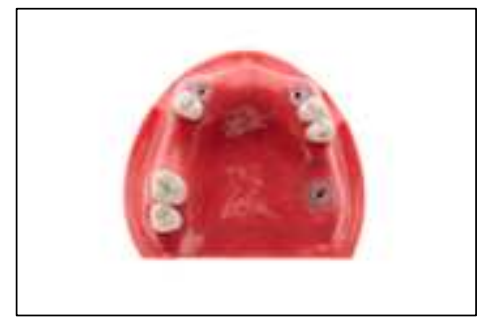

Figure 2. Maxillary typodont duplicated using conventional procedures (CNV group) including polyvinyl siloxane open-tray implant impression technique and poured with Type IV dental stone at room temperature $\left(23^{\circ} \mathrm{C}\right)$.

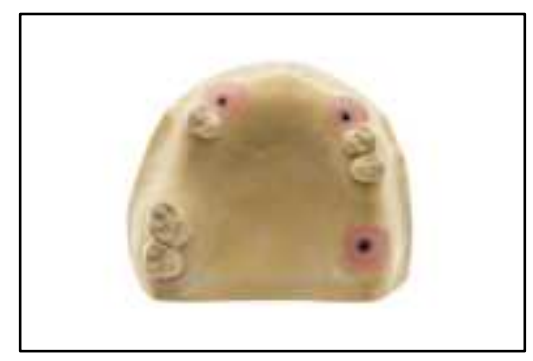

Figure 3. Different scan body designs used to digitize maxillary typodont. A, AM-1 group with 1-piece screw-retained scan body design. B, AM-2 group with 1-piece screw-retained scan body design. C, AM-3 group with 2-piece screw-retained/magnet retained scan body design.
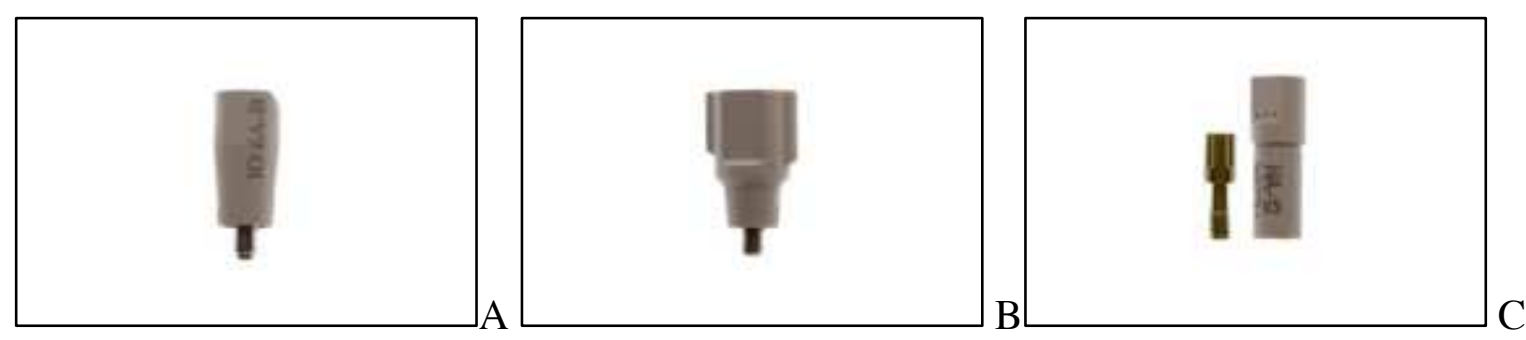
Figure 4. Different digital implant replicas designs positioned on each AM cast. A, AM-1 group with pressure-positioned digital implant replica design. B, AM-2 group with 2-piece screwretained digital implant replica design. C, AM-3 group with 2-piece screw-retained digital implant replica design.
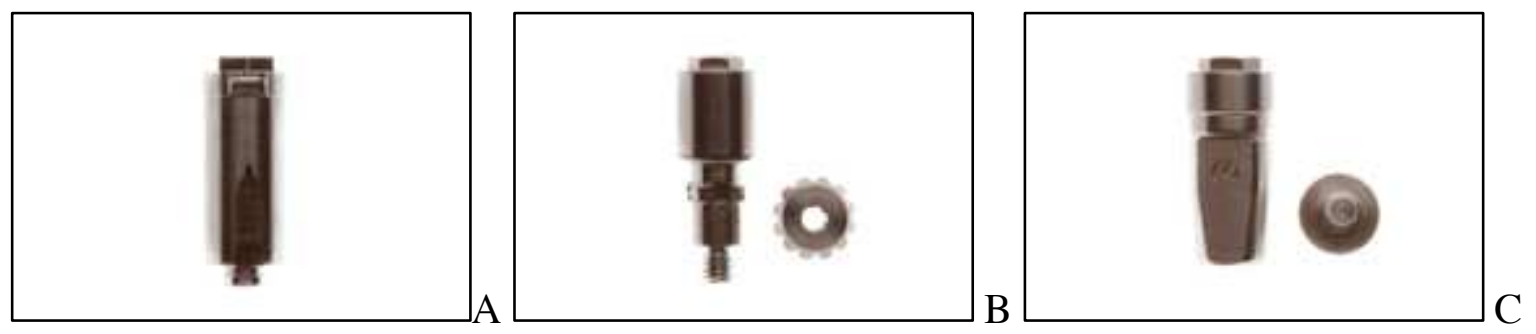

Figure 5. Additively manufactured specimens obtained with polyjet 3D printer (Stratasys J700 Dental; Stratasys). A, AM-1 specimen obtained using ELOS system. B, AM-2 specimen obtained using Nt-Trading system. C, AM-3 specimen obtained using Dynamic Abutment system.
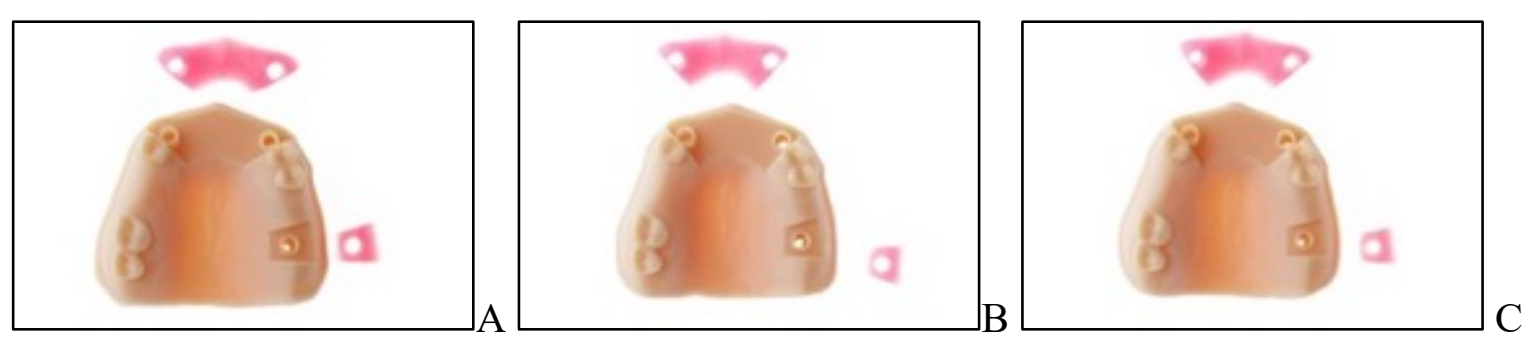

Figure 6. Digital implant replicas positioned on AM specimens. A, AM-1 specimen obtained using ELOS system. B, AM-2 specimen obtained using Nt-Trading system. C, AM-3 specimen obtained using Dynamic Abutment system. 

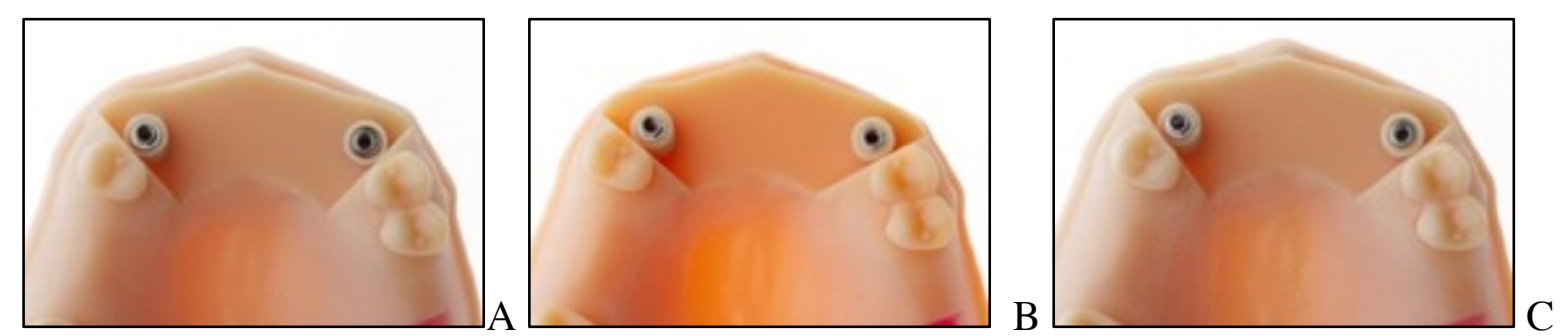

Figure 7. Boxplot of linear discrepancies obtained for CNV, AM-1 (Elos Medtech), AM-2 (NtTrading), and AM-3 (Dynamic abutment) groups. A, X-axis. B, Y-axis. C, Z-axis.
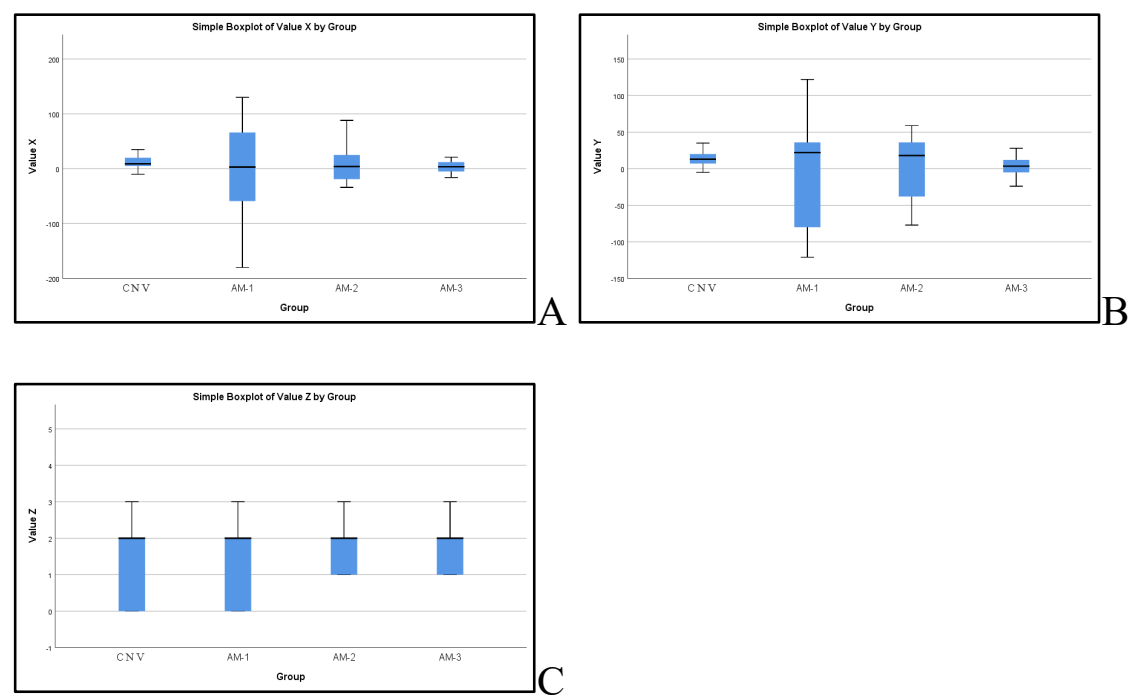

Figure 8. Boxplot of angular discrepancies obtained for CNV, AM-1 (Elos Medtech), AM-2 (NtTrading), and AM-3 (Dynamic abutment) groups. A, Angular discrepancy on XZ projection. B, Angular discrepancy on $\mathrm{YZ}$ projection.
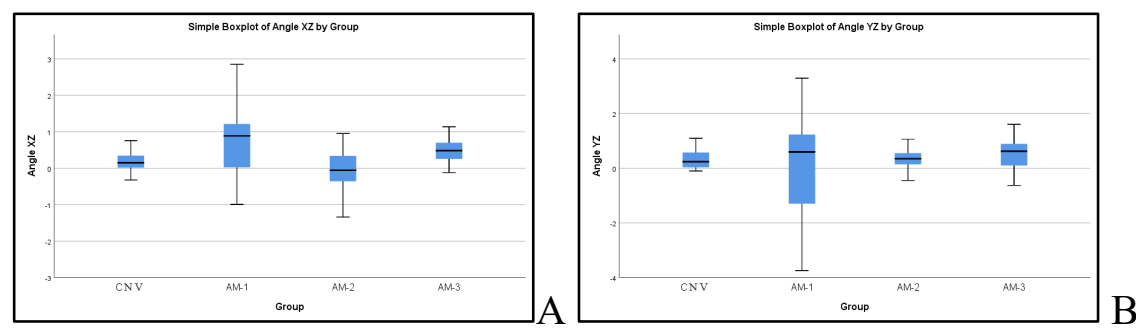
Figure 9. Relationship of different building orientations with digital implant replica position of additively manufactured implant definitive casts.

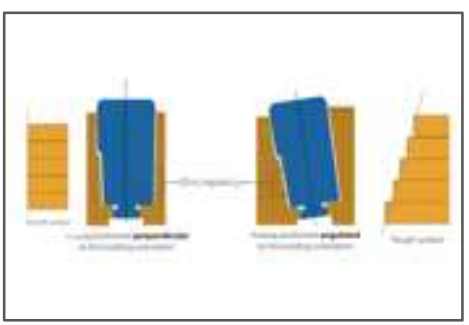

